\title{
Emergency Care of Sepsis in Sub-Saharan Africa: Incidence, Mortality and Non-Physician Clinician Management of Sepsis in rural Uganda from 2010 to 2019
}

\section{Brian Rice ( $\sim$ brice@stanford.edu )}

Stanford University School of Medicine https://orcid.org/0000-0002-9093-1831

\section{Sal Calo}

Cleveland Clinic

John Bosco Kamugisha

Global Emergency Care

Nicholas Kamara

Karoli Lwanga Hospital

Stacey Chamberlain

University of Illinois at Chicago

Global Emergency Care Investigator Study Group n/a Global Emergency Care

\section{Research}

Keywords: Sepsis, Uganda, emergency care, LMIC, mortality, sub-Saharan Africa, fluids, malaria, nonphysician clinicians, task shifting

Posted Date: May 6th, 2021

DOl: https://doi.org/10.21203/rs.3.rs-444837/v1

License: (c) (i) This work is licensed under a Creative Commons Attribution 4.0 International License. Read Full License 


\section{Abstract}

\section{Introduction:}

Little data exists from sub-Saharan Africa describing incidence and outcomes of sepsis in emergency units and uncertainty exists surrounding optimal management of sepsis in low-income settings. There exists limited data regarding quality care metrics for non-physician clinicians trained in emergency care.

\section{Methods:}

Data were obtained for patients seen from 2010-2019 in a rural Ugandan emergency unit staffed by nonphysician clinicians. Sepsis was defined as suspected infection with a qSOFA score $\geq 2$. Descriptive analysis was performed and a multi-variable logistic regression mortality model was created. Analysis included Wilcoxon rank-sum test, t-test, one-way ANOVA, and Fisher's exact test.

\section{Results:}

Overall, 48,653 patient visits from 2010-2019 yielded 17,490 encounters for patients age $\geq 18$ who had suspected infection, including 3,323 with sepsis. Overall sepsis incidence from 2010 to 2019 decreased from $16.4 \%$ to $4.7 \%$, and malarial sepsis incidence decreased from $4.3 \%$ to $0.1 \%$. From 2012 to 2019 , the proportion of septic patients receiving quality care (both fluids and anti-infectives) increased from $36.2 \%$ to $44.7 \%$ but observed mortality rates for non-malarial sepsis increased from $6.3 \%$ to $14.9 \%$ and predicted mortality rates increased from $8.8 \%$ to $12.0 \%$. Higher qSOFA scores were significantly associated with higher rates of both interventions and mortality. All interventions for non-malarial sepsis were independently associated with increased relative risks for death: "fluids alone" RR=1.22 [95\% $\mathrm{Cl} 0.57$ 1.87]; "antibiotics alone" RR=1.25 [95\% Cl 0.60 - 1.91]; "both fluids and antibiotics": $\mathrm{RR}=1.85$ [ $95 \% \mathrm{Cl} 1.02$ $-2.69]$.

\section{Conclusion:}

The largest study of sepsis management and outcomes ever published in both Uganda and sub-Saharan Africa showed decreasing incidence, increasing quality of non-physician clinician care and increasing predicted and observed mortality from 2010 to 2019. With causal associations between antibiotics and mortality deemed implausible, associations between sepsis mortality and interventions likely represent confounding by indication. Defining optimal sepsis care regionally will likely require randomized controlled trials.

\section{Summary Box}

What is already known?

- Sepsis is a major cause of death in Uganda but despite the rapid development of emergency medicine there, the prevalence, outcomes and optimal emergency unit care of sepsis remains unknown as 
emergency medicine is rapidly developing

- Non-physician clinicians provide emergency care in Uganda but the quality and outcomes of their care are unknown

What are the new findings?

- Sepsis represents a large burden of emergency care in rural Uganda in the largest published study of sepsis mortality outcomes published to date in sub-Saharan Africa covering the decade from 2010 2019

- Sepsis and especially malarial sepsis incidence decreased during the study period and non-physician clinicians provided increasingly high quality of care yet sepsis mortality rates increased

- An increased relative risk of death was associated with all sepsis interventions including fluids, antibiotics or both in retrospective multiple variable logistic regression suggesting confounding by indication

What do the new findings imply?

- Emergency unit trends mirror national and regional trends in sepsis over the last decade

- Even large scale retrospective analysis is unable to define optimal sepsis management and prospective randomized controlled trials are necessary

\section{Background}

Sepsis is defined as life-threatening organ dysfunction caused by a dysregulated host response to infection, while septic shock is a subset of sepsis in which there are underlying circulatory, cellular, and metabolic abnormalities that are associated with an even greater risk of mortality.[1] Sepsis is one of the most significant worldwide causes of morbidity and mortality, with 48.9 million annual cases and 11 million annual deaths (19.7\% of all global deaths) according to the most recent analysis of the Global Burden of Disease Study from 2017 data.[2] Sepsis disproportionately affects persons in low-and-middle income countries (LMICs). [2, 3] The World Health Organization has identified sepsis as an international priority, adopting a resolution in 2017 to improve the prevention, diagnosis, and clinical management of sepsis.[4] While in-hospital mortality rates can be greater than $25-30 \%$ across resource settings, mortality rates of up to $38 \%$ are reported in LMICs, particularly in patients who have confirmed bacteremia.[1, 3, 5] In Uganda, a low-income country in sub-Saharan Africa (SSA), the primary causes of death are all infectious in nature - namely malaria, HIV/AIDs, pneumonia, tuberculosis and diarrhea - and all can be associated with sepsis. Inpatient mortality rates have been reported between 34-43\%.[6-9]

Identifying patients with sepsis and septic shock presents a significant challenge. Clinical criteria to identify patients with these syndromes have evolved over time. The most recent Sepsis-3 consensus 
recommendations suggest using bedside clinical criteria, termed the quick Sequential Organ Failure Assessment (qSOFA) score.[1] Adult patients with suspected infection and two or more of these findings are determined to meet sepsis criteria. Using the qSOFA score in a low-resource setting is particularly useful because it does not rely on advanced diagnostic testing. ${ }^{10}$ It is a concise, objective scale which provides opportunity for a rapid objective clinical evaluation of the patient by a variety of providers including nurses, mid-level providers, and physicians.

Management of sepsis globally has changed significantly over the past two decades. In the US and other high-resource settings, "early goal-directed therapy" sparked widespread interest and change in practice patterns for sepsis management, while subsequent trials have called into question the need for higherresourced aspects of the protocol.[10-15] To add to confusion, a landmark study in children in SSA showed that fluid bolus, a cornerstone of management in high resource settings, might actually increase mortality.[16] Some subsequent studies showed similar increased mortality with fluids.[17, 18] However, most experts agree that management should focus early antibiotics and source control plus some combination of fluid resuscitation and early initiation of vasopressor therapy, tailored to the patient presentation.

Emergency medicine is rapidly developing in SSA generally and Uganda specifically.[19-21] With this development, initial sepsis care is shifting to the emergency unit and a better understanding of the burden and outcomes of sepsis from an emergency medicine perspective is required. Sepsis guidelines specific to emergency care in SSA are rare, with the African Federation of Emergency Medicine Handbook serving as the most definitive reference with its recommendations for fluids and early antibiotics following the Surviving Sepsis Campaign.[22] The following analysis utilizes rural Ugandan emergency unit data collected over the decade from 2010 to 2019 and describes sepsis incidence, care and mortality. This evidence base for emergency unit sepsis care will contribute to understanding of larger trends in sepsis incidence over time in Uganda, the ability of non-physician clinician training programmes to impact emergency care quality, and the impact of sepsis management on mortality.

\section{Methods}

\section{Description of Study Site}

All data comes from the emergency unit at Karoli Lwanga Hospital, a rural district hospital located in the town of Nyakibale in the Rukungiri District of southwest Uganda. The hospital has a six-bed emergency unit that opened in 2008 and treats 300 to 700 patients per month arriving between 8:00 am and midnight every day of the year. Since 2010 , the emergency unit has been staffed independently by nonphysician clinicians called emergency care practitioners (ECPs) who received training from physicians working with Global Emergency Care, a Uganda- and US-based non-governmental organization. The ECPs are nurses who have completed a two-year advanced training course in emergency care described in detail elsewhere.[20] While training from 2008 to 2010 was initially supervised by visiting US emergency medicine physicians, ECPs have practiced independent care unsupervised by physicians since 2010 and 
with subsequent training of new cadres in the pilot phase of the project. There is not any real-time physician supervision of clinical care, although the ECPs consult local physicians for patients who require surgery, do not respond to initial treatments, or in whom there is considerable diagnostic uncertainty. Emergency unit patients were admitted to medical and surgical wards staffed by Ugandan physicians with standard levels of training and no connection to Global Emergency Care.

During the study period, the hospital lacked critical care units, ventilators, capabilities for invasive monitoring, and vasopressor medications, other than epinephrine vials for intended management of anaphylaxis. Diagnostic testing was limited to hemoglobin, blood grouping, urinalysis, blood smear for malaria, glucose, cerebrospinal fluid analysis, and HIV rapid testing along with radiology (X-ray and ultrasound) of variable availability. Over time, some additional testing including basic metabolic panel became available and ECPs adopted bedside ultrasonography. Resource utilization by clinicians in this emergency unit is described in detail elsewhere.[23]

Global Emergency Care maintained a prospectively collected quality assurance database of all Karoli Lwanga Hospital emergency unit patient visits, including demographics, vital signs, clinician impression of patient clinical status, medications administered and procedures performed, as well as follow-up vital status (mortality) for all admitted and discharged patients. On Day 3 following initial evaluation in the emergency unit, patients admitted to the hospital were visited in person, while patients discharged from the emergency unit or ward were contacted via phone (if the patient has a phone). A rigorous follow-up protocol which included seven consecutive days of calling patients on the phone before considering them lost to follow-up was used for all patients and this protocol is described in detail elsewhere.[20] When the database was developed, three-day follow-up was chosen to minimize loss to follow-up for admitted and discharged patients in a setting where most patients do not have consistent ability to receive phone calls. Additionally, follow-up after three days was thought to be less reflective of outcomes related to acute care provided in the emergency unit. Ethics approval for the quality assurance database was obtained through the Institutional Review Board at Mbarara University of Science and Technology. Data was input during patient stays in the emergency unit by Global Emergency Care-trained research assistants present in the emergency unit. They used both Microsoft Excel (from 1 January 2010 - 23 March 2012) and Microsoft Access (24 March 2012 - 31 December 2019) databases for data input. Data was abstracted, cleaned, and analyzed by a single researcher (BR) using Stata 16.1 (StataCorp, College Station, TX).

\section{Data Analysis}

Retrospective analysis was performed on prospectively collected data abstracted from the Karoli Lwanga Hospital emergency unit quality assurance database, including all consecutive patients presenting to the emergency unit from January 2010 until December 2019. Suspected infections were defined by a complaint of fever, an objective fever, or a diagnosis consistent with infection (full list of diagnosis is available as Additional file 1 ). Sepsis was defined as a patient with a suspected infection and a qSOFA score of two points or greater with one point each for: tachypnea (respiratory rate $\geq 22$ ); altered 
mentation (defined as a Glasgow Coma Scale $<15$ or an AVPU score other than Alert); hypotension (systolic blood pressure $\leq 100$ ). Hypotension in sepsis was defined as sepsis with a mean arterial pressure less than or equal to 65. After consultation with clinicians, patients without recorded mental status were assumed to have a mental status of "Alert" for analysis, based on practice patterns of typically omitting recording mental status if it was normal. No imputation was otherwise performed for missing data. Malaria was defined as either "smear-positive" (a positive thick/thin smear for Plasmodium falciparum), or "clinical" (patient received diagnosis or treatment of malaria prior to arrival and/or clinical suspicion was high enough despite a negative thick/thin smear). Demographics, vital signs, administration of anti-infectives (including antimalarial, antiviral, antifungal and/or antibiotic medicines) and intravenous fluids (normal saline and/or Ringer's lactate solution), malaria testing (), HIV status (previously known or tested in the emergency unit), and three-day mortality outcomes were analyzed for all patients. Non-parametric age data were compared using the Wilcoxon rank-sum test; continuous variables were compared using the t-test and one-way ANOVA; proportions were compared using Fisher's exact test. A multi-variable logistic regression model to test the significance of associations between independent variables and mortality in septic patients was developed. Area Under Receiver Operating Characteristics Curve (AUROC), Hosmer-Lemeshow Goodness of Fit, and Brier score were all calculated for this model.

\section{Patient and public involvement}

The non-physician clinician training programme was originally developed in response to several years of clinical emergency medicine experience and ongoing health care staffing shortages in Uganda. The positive response of patients, staff and administrators at Karoli Lwanga Hospital to the training programme and their interest in improving patient care led to ongoing research and programme evaluation. Patients and the public were not involved in the design of the study however outcome measures are explicitly patient-oriented. Results will be disseminated through open access publication to allow local clinicians, administrators, policy-makers and researchers to benefit.

\section{Results}

Overall, there were 48,653 patient visits from 2010-2019 (Figure 1). Of these, 17,490 patients were included in the analysis who were aged 18 and older and had a suspected infection. In total, 3,323 patients were defined as having sepsis based on qSOFA scores of 2 or greater. Loss to follow-up analysis was stratified by disposition as the emergency unit overall experienced fundamentally different rates of loss to follow-up for admitted ( $n=702$ of $11,927,5.9 \%$ ) and discharged patients ( $n=2,748$ of $5,403,50.9 \%$ ). Admitted patients were visited in person by research assistants, and follow-up for discharged patients relied on patients owning and answering mobile phones. When comparing septic and non-septic patients by disposition, admitted patients in both groups had similar loss to follow up (5.8\% vs. $6.0 \%, p=0.75)$, while discharged patients with sepsis were less likely to be lost to follow up than non-septic patients $(n=128$ of $340,37.7 \%$ vs. $n=2,620$ of $5,063,51.8 \%, p<0.001)$. The overall mortality rate for combined 
septic and non-septic discharged patients with follow-up was low with only 4 deaths ( $n=4$ of 2,657, $0.15 \%)$ over the 10 years of the study.

The annual number of visits for all patients, patients with suspected infections and with malarial and non-malarial sepsis are displayed in Figure 2. The prevalence of sepsis for patients aged 18 years or older during the study period was $10.4 \%$ for all emergency unit visits. The annual proportion of all visits with suspected infections decreased from $61.7 \%$ in 2010 to $51.0 \%$ in 2019 , with the proportion of those with suspected infections and meeting sepsis criteria decreasing from $16.4 \%$ in 2010 to $4.7 \%$ in 2019 . Nonmalarial sepsis decreased from $12.1 \%$ in 2010 to $4.6 \%$ in 2019. Malarial sepsis (combined "smearpositive" and "clinical") had an even more pronounced decrease from its peak of $6.5 \%$ in 2013 to $0.3 \%$ in 2019. The proportions in Figure 2 were calculated using all emergency unit patient visits as a denominator $(n=31,856)$ to describe overall emergency unit trends. All subsequent analyses were restricted to patients with suspected infection $(n=17,490)$.

Characteristics of septic and non-septic patients with suspected infection were compared (Table 1).

Table 1: Characteristics of Non-Septic (qSOFA<2) and Septic (qSOFA $\geq 2$ ) Emergency Unit Patients With Suspected Infections $(N=17,490)$ 


\begin{tabular}{|c|c|c|c|}
\hline & $\begin{array}{c}\text { No Sepsis (qSOFA < 2) } \\
n=14,167\end{array}$ & $\begin{array}{c}\text { Sepsis (qSOFA } \geq 2 \text { ) } \\
n=3,323\end{array}$ & p-Value \\
\hline Age, median (IQR) & $43(26-68)$ & $40(27-60)$ & $<0.001^{\dagger}$ \\
\hline \multicolumn{4}{|l|}{ Age Group } \\
\hline 18-64 years old, total (\%) & $10086(71.2)$ & $2642(79.5)$ & $<0.001$ \\
\hline $65+$ years old, total $(\%)$ & $3991(28.1)$ & $661(19.9)$ & $<0.001$ \\
\hline Female, total (\%) & $7327(51.8)$ & $1803(54.3)$ & 0.009 \\
\hline Systolic Blood Pressure, mean (95\% CI) & $119.5(119.2-119.9)$ & $93.6(93.1-94.1)$ & $<0.001^{\dagger \dagger}$ \\
\hline Heart Rate, mean $(95 \% \mathrm{CI})$ & $92.1(91.8-92.5)$ & $105.1(104.2-105.9)$ & $<0.001^{\dagger \dagger}$ \\
\hline Respiratory Rate, mean (95\% CI) & $21.9(21.8-22.0)$ & $28.8(28.6-29.1)$ & $<0.001^{\dagger \dagger}$ \\
\hline Oxygen Saturation, mean $(95 \% \mathrm{CI})$ & $95.3(95.2-95.4)$ & $92.4(92.1-92.7)$ & $<0.001^{\dagger \dagger}$ \\
\hline \multicolumn{4}{|l|}{ qSOFA Criteria } \\
\hline Respiratory rate $\geq 22$ breaths per minute, $n$ (\%) & $4457(31.5)$ & $3207(96.5)$ & $<0.001$ \\
\hline Systolic blood pressure $\leq 100 \mathrm{mmHg}, \mathrm{n}$ (\%) & $837(5.9)$ & $1532(46.1)$ & $<0.001$ \\
\hline Altered mentation (GCS $<15$ or AVP $\neq \mathrm{A}$ ), $\mathrm{n}$ (\%) & $244(1.7)$ & $441(13.3)$ & $<0.001$ \\
\hline \multicolumn{4}{|l|}{ Co-existing Infections } \\
\hline Malaria: Smear-Positive, n (\%) & $2223(15.7)$ & $690(20.8)$ & $<0.001$ \\
\hline Malaria: Clinical, n (\%) & $1880(13.3)$ & $467(14.1)$ & 0.24 \\
\hline HIV, n (\%) & $1266(8.9)$ & $708(21.4)$ & $<0.001$ \\
\hline \multicolumn{4}{|l|}{ Clinician Impression, $\mathrm{n}(\%)$} \\
\hline "Not Sick" & $7753(55.1)$ & $951(28.8)$ & $<0.001$ \\
\hline "Sick" & $6109(43.4)$ & $2178(66.0)$ & $<0.001$ \\
\hline "Toxic" & $211(1.5)$ & $171(5.2)$ & $<0.001$ \\
\hline \multicolumn{4}{|l|}{ Disposition, $\mathbf{n}(\%)$} \\
\hline Admitted & $8999(63.5)$ & $2927(88.1)$ & $<0.001$ \\
\hline Discharged & $5063(35.7)$ & $340(10.2)$ & $<0.001$ \\
\hline Expired in ED & $47(0.3)$ & $35(1.1)$ & $<0.001$ \\
\hline Operating Theater & $58(0.4)$ & $21(0.6)$ & 0.11 \\
\hline
\end{tabular}

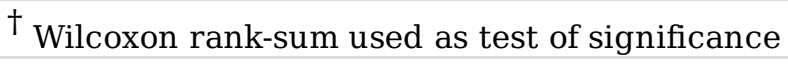

t† $\mathrm{T}$-test used as test of significance

All others use Fisher's exact test as test of significance

There were significant differences between the groups, with septic patients being younger, more likely to be female, more likely to have abnormalities in all vital signs (blood pressure, heart rate, respiratory rate, oxygen saturation, mental status), more likely to be coinfected both with HIV and malaria, more likely to have a clinician impression of "Serious" or "Critical" illness, and more likely both to be admitted to the hospital and to die in the emergency unit. Levels of missing data were generally low: age $(n=110,0.63 \%)$, gender $(n=12,0.07 \%)$, blood pressure $(n=681,3.89 \%)$, heart rate $(n=515,2.94 \%)$, respiratory rate $(n=1,460$, $8.35 \%)$, oxygen saturation ( $n=1,338,7.65 \%)$, and clinician impression of illness severity $(n=118,0.67 \%)$. Mental status was an exception with a high level of missing data $(n=10,720,62.7 \%)$. 
The mortality and interventions in patients stratified by qSOFA score are displayed in Table 2 below. Interventions were incompletely captured for the 3,942 patients in the original Excel database from 2010 - 2012, therefore analysis of interventions in sepsis over time was restricted to the 13,548 patients recorded in the Access database from 2012 - 2019. Increasing qSOFA scores were associated with higher rates of death and higher rates of receiving both fluids and anti-infectives. The dichotomous splitting of patients into "No Sepsis" and "Sepsis" using the cutoff of qSOFA $\geq 2$ shows the same clinically and statistically significant associations.

Table 2: Interventions and mortality for patients 2012-2019 stratified by qSOFA score $(n=13,549)$

\begin{tabular}{|c|c|c|c|c|c|c|c|c|}
\hline & \multicolumn{5}{|c|}{ qSOFA Score } & \multicolumn{3}{|c|}{ Dichotomous qSOFA Score } \\
\hline \multirow{4}{*}{$\begin{array}{l}\text { Total Cases, } \mathrm{n} \\
\text { Total Deaths, } \mathrm{n} \\
\text { Crude Mortality Rate, \% } \\
\quad[95 \% \mathrm{CI}]\end{array}$} & Zero & One & Two & Three & $\mathrm{p}$-Value & $\begin{array}{c}\text { No } \\
\text { Sepsis } \\
(<2)\end{array}$ & $\begin{array}{l}\text { Sepsis } \\
(\geq 2)\end{array}$ & $\mathrm{p}$-Value \\
\hline & 5855 & 5331 & 2229 & 133 & \multirow{4}{*}{$\begin{array}{c}\mathrm{n} / \mathrm{a} \\
\mathrm{n} / \mathrm{a} \\
<0.001^{\dagger}\end{array}$} & 11186 & \multirow{4}{*}{$\begin{array}{c}2362 \\
216 \\
9.1[8.0 \\
-10.3]\end{array}$} & \multirow{4}{*}{$\begin{array}{c}\mathrm{n} / \mathrm{a} \\
\mathrm{n} / \mathrm{a}\end{array}$} \\
\hline & 65 & 215 & 189 & 27 & & 280 & & \\
\hline & $\begin{array}{c}1.1[0.9 \\
-1.4]\end{array}$ & $\begin{array}{c}4.0[3.5 \\
-4.6]\end{array}$ & $\begin{array}{c}8.5[7.4 \\
-9.7]\end{array}$ & $\begin{array}{c}20.3[13.8 \\
-28.1]\end{array}$ & & $\begin{array}{c}2.5[2.2 \\
-2.8]\end{array}$ & & \\
\hline $\begin{array}{l}\text { Proportion receiving } \\
\text { interventions, (\%) }\end{array}$ & & & & & & & & \\
\hline $\begin{array}{l}\text { Neither fluids nor anti- } \\
\text { infectives }\end{array}$ & $\begin{array}{l}3565 \\
(60.9)\end{array}$ & $\begin{array}{c}2168 \\
(40.7)\end{array}$ & $\begin{array}{c}505 \\
(22.7)\end{array}$ & $18(13.5)$ & \multirow{3}{*}{$\begin{array}{c}0.08^{\dagger} \\
0.009^{\dagger} \\
0.004^{\dagger}\end{array}$} & $\begin{array}{c}5733 \\
(51.3)\end{array}$ & $\begin{array}{c}523 \\
(22.1)\end{array}$ & $<0.001^{* *}$ \\
\hline Fluids only & $\begin{array}{c}837 \\
(14.3)\end{array}$ & $\begin{array}{l}1092 \\
(20.5)\end{array}$ & $\begin{array}{c}528 \\
(23.7)\end{array}$ & $29(21.8)$ & & $\begin{array}{c}1929 \\
(17.2)\end{array}$ & $\begin{array}{c}557 \\
(23.6)\end{array}$ & $<0.001^{* *}$ \\
\hline Anti-infectives only* & $\begin{array}{c}794 \\
(13.6)\end{array}$ & $\begin{array}{c}966 \\
(18.1)\end{array}$ & $\begin{array}{c}380 \\
(17.1)\end{array}$ & $16(12.0)$ & & $\begin{array}{c}1760 \\
(15.7)\end{array}$ & $\begin{array}{c}396 \\
(16.8)\end{array}$ & $0.22^{* *}$ \\
\hline $\begin{array}{l}\text { Both fluids and anti- } \\
\text { infectives }\end{array}$ & $\begin{array}{c}659 \\
(11.3)\end{array}$ & $\begin{array}{l}1105 \\
(20.7)\end{array}$ & $\begin{array}{c}816 \\
(36.6)\end{array}$ & $70(52.6)$ & $0.06^{\dagger}$ & $\begin{array}{c}1764 \\
(15.8)\end{array}$ & $\begin{array}{c}886 \\
(37.5)\end{array}$ & $<0.001 * *$ \\
\hline
\end{tabular}

$\dagger$ ANOVA used as test of significance

${ }^{\dagger \dagger}$ T-test used as test of significance

* Anti-infectives include antibiotics, antivirals and/or antimalarials

** Fisher's exact test used as test of significance

Figure 3 displays the annual trends in sepsis management and mortality from 2012-2019. The rates of receiving both fluids and anti-infectives increased (36.2\% in 2012 to $44.7 \%$ in 2019), while the rates of receiving neither fluids nor anti-infectives in the emergency unit decreased $(25.4 \%$ in 2012 to $13.8 \%$ in 2019). (Figure 3a) Over that same time period, sepsis mortality increased (6.2\% in 2012 to $14.5 \%$ in 2019), while non-sepsis mortality remained stable (2.7\% in 2012 to $2.2 \%$ in 2019).(Figure 3b) Mortality data for 2010 and 2011 are not included in Figure 3b for consistency with Figure 3a and are as follows: in 2010 , non-sepsis mortality was $3.3 \%$ [ $95 \% \mathrm{Cl} 2.2-4.4]$ and sepsis mortality was $7.6 \%[95 \% \mathrm{Cl} 4.9-$ 
10.3]; in 2011 non-sepsis mortality was $2.6 \%$ [ $95 \% \mathrm{Cl} 1.8-3.4]$ and sepsis mortality was $6.8 \%$ [ $95 \% \mathrm{Cl} 4.5$ $-9.1]$.

Figure 4 displays trends in prevalence and associated mortality for sub-groups of septic patients by year from 2010-2019. Across programme years, septic patients are more likely to be elderly and to have qSOFA scores of 3 (both higher mortality sub-groups), less likely to have malaria (a lower mortality sub-group) and equally likely to have hypotension (a much higher mortality sub-group).

To understand the relative impact of these changes in both septic patients and sepsis care, a multivariable logistic regression model was created for septic patients. Patients with complete treatment data from $2012-2019$ both with malaria $(n=741)$ and without malaria $(n=1621)$ were separated to identify independent predictors of mortality. Looking at malarial sepsis only, there were 54 deaths $(7.3 \%$ mortality rate) in the 741 cases from 2012-2019. Further analysis of this data over time was limited by the precipitous drop in malaria incidence and deaths culminating in the years 2017-2019 having 31, 26 and 11 cases and two, three and one deaths respectively. Analysis of the impact of treatments was limited by the baseline group of "No treatment received in ED" having only one death in 156 cases, thus making comparisons unusably imprecise. Consequently, malaria was excluded from subsequent logistic regression analysis in this manuscript but is available as Additional file $\mathbf{2}$.

Table 3: Logistic Regression Model of Mortality in Septic (qSOFA $\geq 2$ ) Patients Without Malaria: $2012-2019(N=1,621)$ 


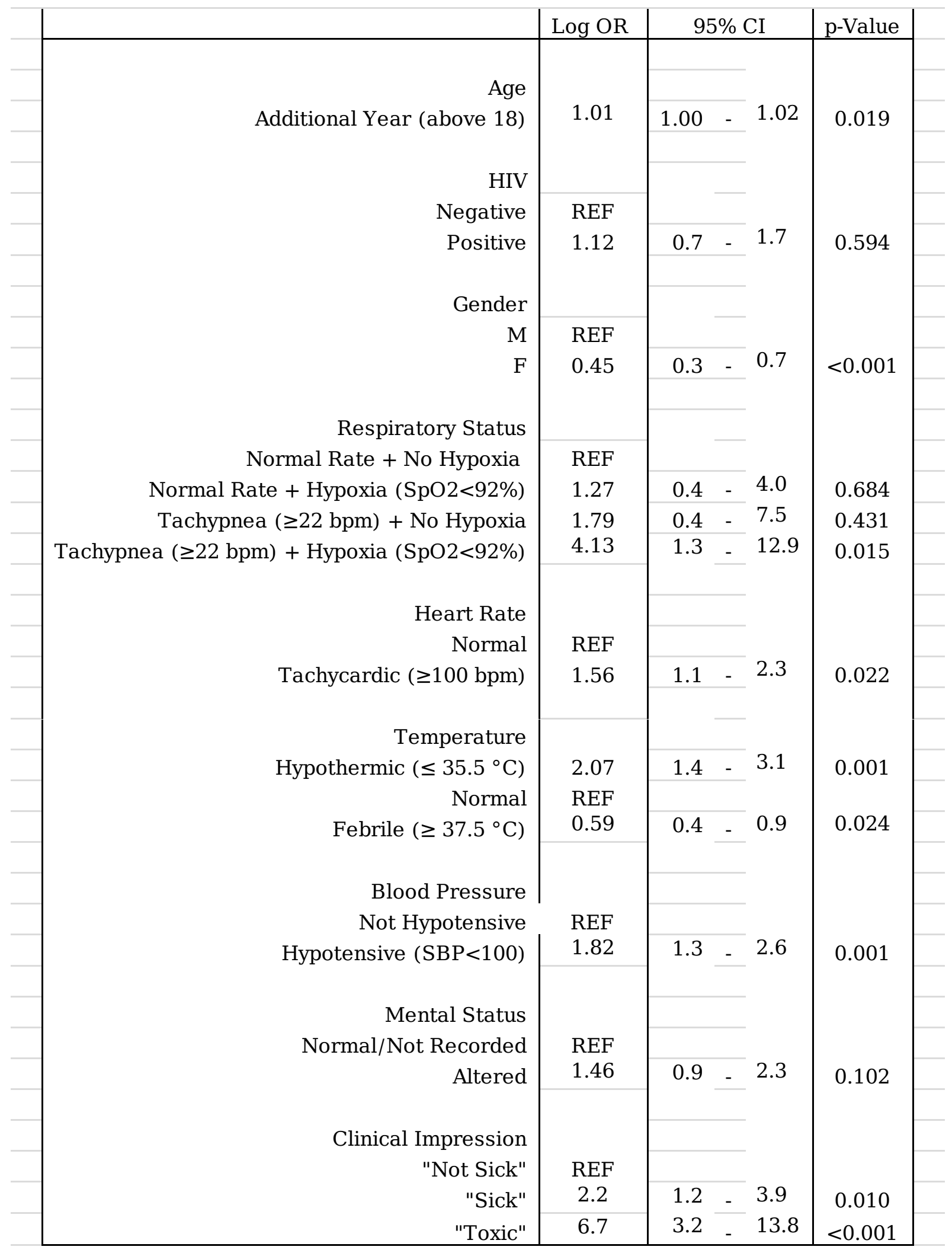

Looking at the multiple variable logistic regression model for non-malarial sepsis mortality (Table 3), the odds ratio (OR) of death was significantly increased seen in patients with advancing age, hypothermia, hypotension, tachycardia, with both hypoxia and tachypnea, and for patients with concerning clinical condition upon arrival ("Sick" and "Toxic"). There was a significantly reduced OR of death for patients 
who were female or febrile. The $p$-value for the Hosmer-Lemeshow goodness of fit test was 0.10 , the Brier score was 0.079 , and the AUROC was $0.80(95 \% \mathrm{Cl} 0.77-0.83)$. The predicted mortality coefficients generated by this model were plotted against observed mortality across programme years and both predicted and observed mortality could be seen to increase across the study period (Figure 5).

The categorical variable for sepsis treatment in the emergency unit displayed visually in Figure $\mathbf{3 a}$ above (no treatment in the emergency unit, fluids alone, antibiotics alone, or both fluids and antibiotics) was added to the logistic regression model above to assess the independent association between treatments and mortality in all septic patients without malaria. All treatments for sepsis were associated with increased relative risk (RR) of death as compared to "no treatment in the emergency unit" after controlling for the variables in the logistic regression model above. The RR were as follows: "fluids alone" RR=1.22 [95\% Cl 0.57 - 1.87]; "antibiotics alone" RR=1.25 [95\% Cl 0.60 - 1.91]; "both fluids and antibiotics":

$\mathrm{RR}=1.85[95 \% \mathrm{Cl} 1.02-2.69]$. Only the RR associated with administration of "both fluids and antibiotics" reached statistical significance.

\section{Discussion}

The analysis presented above describes a decade of Ugandan emergency unit experience with sepsis management and outcomes. The 3323 patients included in this analysis represent the largest study of sepsis outcomes in SSA to date, including more patients than the 2800 patients in all other published studies of sepsis outcomes in SSA combined.[24] The number of subjects alone makes the study noteworthy as does the longitudinal nature of the data providing insight into the changing landscape of sepsis and emergency care in Uganda over the last decade. Additionally, this analysis provides strong evidence that an emergency care training programme for non-physician clinicians can produce and sustain improvements in care quality as defined by adherence to expert recommendations at the time.

Sepsis was a substantial burden in this Ugandan emergency unit over a decade with $10.4 \%$ of all adult emergency unit visits meeting criteria for sepsis. However, stratifying sepsis incidence by year showed a marked decrease from $16.5 \%$ of all patients seen in 2010 to $4.8 \%$ in 2019. Though both overall sepsis and non-malarial sepsis decreased over time, the drop in malarial sepsis incidence was profound: $4.3 \%$ in 2010 to $0.1 \%$ in 2019 . These findings are in accord with the observed decrease in malaria as a cause of death and disability in Uganda over the period of 2007-2017 in the Global Burden of Diseases, Injuries and Risk Factors (GBD) survey. ${ }^{6}$ The National Malaria Control Programme in Uganda which was active during the study period, employed a combination of control measures including long-lasting insecticidal nets, indoor residual spraying, and intermittent preventive treatment for malaria during pregnancy and its success is the likely cause of the observed reduction in emergency unit burden. ${ }^{29}$ The sharp downward trend in non-malarial sepsis and sepsis overall seen here has also been observed during the study period in national and regional level analyses of GBD data. $[2,25]$ While GBD data is not specific to the emergency unit, the few emergency medicine-focused studies from SSA show a similar downward trend in sepsis incidence.[26] The ultimate causes of this trend are likely tied to overall economic and health 
systems development in Uganda and throughout SSA in general, but detailed analysis of the impact of those forces on emergency unit malaria incidence and mortality is outside the scope of this manuscript.

The data presented in Table 2 showed that qSOFA performed well in classifying patients with higher and lower mortality rates in line with other research suggesting the value of qSOFA in SSA.[27] There was clear identification of increased mortality not just between septic/non-septic patients (qSOFA $<2$ and $\geq 2$ ), but also an increase of crude mortality as qSOFA scores increased from zero to three. Overall this analysis both contributes to support for qSOFA in low-income, SSA emergency unit settings and represents the first study to the authors' knowledge that demonstrates the potential utility of qSOFA in a setting where clinical care is provided by non-physician clinicians. Further prospective studies would be needed to fully validate the utility of qSOFA by non-physician clinicians.

Non-physician clinician sepsis care quality also improved significantly during the study period (as depicted in Table 3a). The non-physician clinician training employed in the study setting promotes ongoing practice improvement and has focused on increasing the rates of early resuscitation of septic patients in accordance with regional guidelines for emergency care.[22] The rates of fluid and antibiotic administration provided by non-physician clinicians compared favorably to the standard of care provided by admitting medical officers in other Ugandan hospitals during the same time period.[9] Some authors have suggested that improved training of nurses, paramedical assistants, and other non-physician clinicians could significantly improve sepsis identification and management and can be done at low cost. $[6,28]$ Taking sepsis care as a reasonable proxy for emergency care in general, the findings of this manuscript have clear policy implications: emergency medicine training programmes for non-physician clinicians produce a workforce capable of delivering quality care, as defined by meeting published care guidelines. Such programmes may address both emergency care staffing shortages in SSA and help meet the pressing global development goals of improving quality of care.[29]

Despite the increasing quality of care, observed mortality for septic patients increased across the study period while mortality for non-septic patients did not (as seen in Table 3b). However, the prevalence of higher and lower mortality subgroups of septic patients also changed substantially over time, which was hypothesized to have changed the predicted mortality over time (as seen in Figure 4). When both predicted and observed mortality were plotted over time in Figure 5, it was evident that both did in fact increase over time. Given the global trends towards reduced sepsis incidence and mortality cited above, this was somewhat surprising. One possible explanation is "self-triage" or the decisions made by patients about where to seek emergency care for themselves or their family. Self-triage can be an important factor in many settings and the local providers and patients have given feedback to the development programme for many years the local community has viewed the emergency unit positively since it was established in 2008. One interpretation of the gradually increasing predicted mortality across programme years is that the local community's increasing confidence in the emergency unit over the years has led increasingly sick patients to preferentially seek care there. This interpretation is supported by the data displayed in Figure 4 showing a marked increase in the proportions of patients in high-mortality 
subgroups (e.g. elderly, qSOFA=3) over time. While these variables were included in the overall mortality model, further analysis of this phenomena is outside the scope of this manuscript.

One variable not commonly included in mortality models - but which independently predicted increased mortality in this model - was the initial "clinical impression" of patients upon their arrival to the emergency unit. The training programme has long stressed that the clinical skill of rapidly identifying both critically-ill and generally well-appearing patients is a cornerstone of emergency care. Every nonclinician trained by the programme was taught to categorize patients as "not sick", "sick" or "toxic" early in their evaluation and prioritize interventions based on this assessment. The strongly significant independent association of this variable with mortality ("Sick" OR $=2.2,95 \% \mathrm{Cl} 1.2-3.9$; "Toxic" OR = 6.7, $95 \% \mathrm{Cl} 3.2$ - 13.8) even when controlling for comorbidities, age and vital sign abnormalities suggests that this type of clinical assessment has utility in addition to other objective data in the emergency unit. The ability of emergency care-trained non-physician clinicians to utilize this skill central to emergency medicine physician training to reliably identify patients at higher risk for short-term mortality contributes to the overall argument for their clinical capacity to evaluate and provide unsupervised emergency care for undifferentiated septic patients.

Despite the clearly increasing adherence to quality care guidelines across time (as defined by providing fluids and anti-infectives in the emergency unit and depicted in Figure 3a) there was no trend towards reducing observed versus predicted mortality and in fact both observed and predicted mortality increased during the study period (Figure 3b, Figure 5). Furthermore, the logistic regression model including these interventions showed increased mortality with all sepsis interventions and requires additional investigation. This apparent harm is noteworthy as optimal sepsis resuscitation in SSA remains controversial with a recent review of sepsis guidelines in SSA citing multiple studies showing harm from fluids. $[16,18,30,31]$ Retrospective analysis such as that used in this study is challenged by one of the first principles of emergency medicine: the most critically-ill patients with the highest mortality rates receive the most interventions. Table 2 provides supporting data for the existence of this care pattern by demonstrating significant associations between increasing interventions, increasing mortality and increasing qSOFA scores. This practice pattern ultimately creates substantial confounding by indication in associating interventions with mortality without clear causation.

The logistic regression model for mortality was developed in an attempt to control for confounding by indication by including not only objective data (e.g., vital signs) but also subjective data (e.g., clinical impression). It was hoped that the inclusion of subjective evaluation would control for the recognition of critical illness by the providers and their associated decisions to escalate interventions. However, this model ultimately showed a clinically but not statistically significant association between mortality and either fluids ( $R R=1.22,95 \% \mathrm{Cl} 0.57-1.87$ ) or antibiotics ( $R R=1.25,95 \% \mathrm{Cl} 0.60-1.91)$ given singly, and a clinically and statistically significant association between mortality and fluids and antibiotics given together $(R R=1.85,95 \% \mathrm{Cl} 1.02-2.69)$. No literature published to date suggests that antibiotics increase mortality in sepsis in any setting. Therefore, their association with increased mortality functions as a falsification test arguing that the model was unable to adequately control for confounding and arguing 
against drawing any conclusions regarding causative associations between treatments for sepsis and mortality in this model. Different analytic approaches may provide additional answers but, given that this manuscript includes a decade of data and more septic patients than exist in all other studies of sepsis outcomes in SSA combined, retrospective analysis may be inadequate to define optimal sepsis care in Uganda or SSA due to this overwhelming confounding by indication. As health systems and emergency care rapidly develop in Uganda and SSA more generally, they do so without the necessary evidence base to guide optimal sepsis care. Controlled, prospective, randomized clinical trials are needed to isolate the mortality impact of individual sepsis treatments and/or care bundles for emergency unit patients in these settings.

\section{Limitations}

There are several limitations for this study. The first limitations are those of the registry database. Loss to follow-up for discharged patients was high, despite rigorous methods that included calling patients every day for seven consecutive days following discharge. However, the large number of patients that did not have phones or answer their phones limited response rates. Despite mortality being exceedingly low at $0.15 \%$ over 10 years of discharged patients, this low response rate likely resulted in underestimating discharged mortality. Multiple imputation of mortality data to control for this was considered but deemed methodologically unsound. Secondly, the registry data are limited to a single center. The regional specificity of causes and management of sepsis may limit the generalizability of the findings above. Mortality reported in this study was lower overall than in similar settings. This may be due to larger trends in mortality in the region but also likely reflects limitations of the emergency unit registry data which only recorded vital status at three days and therefore may underestimate longer-term measures such as total in-hospital mortality. Unique patient visits were recorded and reported instead of unique patients which may impact overall incidence of sepsis. As discussed above, logistic regression models were likely limited in their ability to control for confounding by indication. Limitations in data prevented inclusion weightbased dosing of fluids and appropriateness of anti-infectives as variables in analysis. Future randomized prospective studies may benefit from looking specifically at sepsis outcomes related to types of antiinfectives and volume of fluids administered. Missing data regarding mental status (omitted in over $60 \%$ of charts) may have systematically under-recognized sepsis based on qSOFA.

\section{Conclusions}

Data for 3,323 septic patients from 2010-2019 at a single rural Ugandan emergency unit staffed by nonphysician clinicians were included in retrospective analysis and represent the largest study of sepsis outcomes in SSA published to date. The annual proportions of patients with sepsis decreased over time, and the non-physician clinicians providing emergency care significantly improved their adherence to sepsis guidelines. These findings provide insight into how sepsis in Ugandan emergency medicine reflects sepsis trends at national and regional levels and demonstrate that non-physician clinicians are both capable of delivering high-quality emergency care in SSA and represent a possible solution to 
ongoing healthcare staffing shortages. As the nature of patients seeking emergency sepsis care changed over time the expected mortality rates increased but, despite improvements in care quality, the observed mortality rates also increased. Ultimately, logistic regression models were unable to control for confounding and isolate the mortality impact of fluids and/or antibiotics in sepsis. With retrospective analysis unable to address ongoing concerns about the safety of fluids in sepsis in SSA and emergency medicine developing rapidly in both Uganda and SSA, randomized controlled trials are urgently needed to define and guide optimal sepsis management.

\section{Abbreviations}

$\mathrm{Cl}$ - confidence interval

ECP - emergency care practitioner

LMICs - low- and middle-income countries

$\mathrm{OR}$ - odds ratio

qSOFA - quick Sequential Organ Failure Assessment

$\mathrm{RR}$ - relative risk

SSA - Sub-Saharan Africa

\section{Declarations}

Ethics approval and consent to participate

Ethics approval for the quality assurance emergency unit database was obtained through the Institutional Review Board at Mbarara University of Science and Technology and University of Massachusetts.

Consent for publication (individual)

Not applicable.

Availability of data and materials

The datasets used and analyzed during the current study are available from the corresponding author on reasonable request.

Competing interests

The authors declare no competing interests.

Funding 
This research received no specific grant from any funding agency in the public, commercial or not-forprofit sectors. No funding was received for the development or analysis of the database. Paid stipends for research assistants and a programme director were provided partially by Global Emergency Care. Salary support for Emergency Care Practitioners/non-physician clinicians was also provided partially by Global Emergency Care.

\section{Acknowledgements}

The authors would like to acknowledge the indispensable contributions of the Global Emergency Care Investigator Study Group including Mark Bisanzo, Heather Hammerstedt, and Bradley Dreifuss.

\section{Author's contributions}

BR, S. Calo and S. Chamberlain contributed to study concept and design. BR, JBK, NK conducted data acquisition. BR cleaned data, planned statistical analysis, and performed statistical analysis. BR, S. Calo and S. Chamberlain contributed to data interpretation. BR and S. Calo primarily wrote the manuscript with revisions and contributions from S. Chamberlain, JBK, and NK. Global Emergency Care Investigator Group were involved in data acquisition and study concept. All authors provided edits and final approval of the manuscript.

\section{References}

1. Singer M, Deutschman CS, Seymour CW, Shankar-Hari M, Annane D, Bauer M, et al. The Third International Consensus Definitions for Sepsis and Septic Shock (Sepsis-3). JAMA. 2016;315:80110. doi:10.1001/jama.2016.0287.

2. Rudd KE, Johnson SC, Agesa KM, Shackelford KA, Tsoi D, Kievlan DR, et al. Global, regional, and national sepsis incidence and mortality, 1990-2017: analysis for the Global Burden of Disease Study. The Lancet. 2020;395:200-11. doi:10.1016/S0140-6736(19)32989-7.

3. Fleischmann C, Scherag A, Adhikari NKJ, Hartog CS, Tsaganos T, Schlattmann P, et al. Assessment of Global Incidence and Mortality of Hospital-treated Sepsis. Current Estimates and Limitations. Am J Respir Crit Care Med. 2016;193:259-72.

4. World Health Organization. Improving the prevention, diagnosis and clinical management of sepsis (WHA70.7). 2017; Bills/Resolutions. https://www.who.int/servicedeliverysafety/areas/sepsis/en/.

5. Becker JU, Theodosis C, Jacob ST, Wira CR, Groce NE. Surviving sepsis in low-income and middleincome countries: new directions for care and research. Lancet Infect Dis. 2009;9:577-82.

6. Rudd KE, Tutaryebwa LK, Eoin West T. Presentation, management, and outcomes of sepsis in adults and children admitted to a rural Ugandan hospital: A prospective observational cohort study. 2017; Generic. doi:10.1371/journal.pone.0171422.

7. Institute for Health Metrics and Evaluation (IHME). GBD Compare Data Visualization. IHME Website. 2020. http://vizhub.healthdata.org/gbd-compare. 
8. Amir A, Saulters KJ, Olum S, Pitts K, Parsons A, Churchill C, et al. Outcomes of patients with severe sepsis after the first 6 hours of resuscitation at a regional referral hospital in Uganda. $J$ Crit Care. 2016;33 Journal Article:78-83.

9. Jacob ST, Banura P, Baeten JM, Moore CC, Meya D, Nakiyingi L, et al. The impact of early monitored management on survival in hospitalized adult Ugandan patients with severe sepsis: a prospective intervention study*. Crit Care Med. 2012;40:2050-8.

10. Rivers E, Nguyen B, Havstad S, Ressler J, Muzzin A, Knoblich B, et al. Early Goal-Directed Therapy in the Treatment of Severe Sepsis and Septic Shock. NEnglJMed. 2001;345:1368-77. doi:10.1056/NEJMoa010307.

11. The ProCESS Investigators. A Randomized Trial of Protocol-Based Care for Early Septic Shock. New England Journal of Medicine. 2014;370:1683-93. doi:10.1056/NEJMoa1401602.

12. The ARISE Investigators and the ANZICS Clinical Trials Group. Goal-Directed Resuscitation for Patients with Early Septic Shock. New England Journal of Medicine. 2014;371:1496-506. doi:10.1056/NEJMoa1404380.

13. Mouncey PR, Osborn TM, Power GS, Harrison DA, Sadique MZ, Grieve RD, et al. Trial of Early, GoalDirected Resuscitation for Septic Shock. New England Journal of Medicine. 2015;372:1301-11. doi:10.1056/NEJMoa1500896.

14. The PRISM Investigators. Early, Goal-Directed Therapy for Septic Shock - A Patient-Level MetaAnalysis. New England Journal of Medicine. 2017;376:2223-34. doi:10.1056/NEJMoa1701380.

15. Rhodes A, Evans LE, Alhazzani W, Levy MM, Antonelli M, Ferrer R, et al. Surviving Sepsis Campaign: International Guidelines for Management of Sepsis and Septic Shock: 2016. Intensive Care Med. 2017:43:304-77.

16. Maitland K, Kiguli S, Opoka RO, Engoru C, Olupot-Olupot P, Akech SO, et al. Mortality after Fluid Bolus in African Children with Severe Infection. The New England Journal of Medicine. 2011;364:2483-95. doi:10.1056/NEJMoa1101549.

17. Morton B, Stolbrink M, Kagima W, Rylance J, Mortimer K. The Early Recognition and Management of Sepsis in Sub-Saharan African Adults: A Systematic Review and Meta-Analysis. International journal of environmental research and public health. 2018;15:2017. doi:10.3390/ijerph15092017.

18. Andrews B, Semler MW, Muchemwa L, Kelly P, Lakhi S, Heimburger DC, et al. Effect of an Early Resuscitation Protocol on In-hospital Mortality Among Adults With Sepsis and Hypotension: A Randomized Clinical Trial. JAMA. 2017;318:1233-40.

19. Humphreys G. Improving emergency care in Uganda. Bull World Health Organ. 2019;97:314-5. doi:10.2471/BLT.19.020519.

20. Hammerstedt H MD, MPH, Maling S MBChB, Kasyaba R MBChB, Dreifuss B MD, Chamberlain S MD, MPH, Nelson S MD, et al. Addressing World Health Assembly Resolution 60.22: A Pilot Project to Create Access to Acute Care Services in Uganda. Annals of Emergency Medicine. 2014;64:461-8. doi:10.1016/j.annemergmed.2014.01.035. 
21. Obermeyer Z, Abujaber S, Makar M, Stoll S, Kayden SR, Wallis LA, et al. Emergency care in 59 lowand middle-income countries: a systematic review. Bulletin of the World Health Organization. 2015;93:577-586G. http://www.who.int/entity/bulletin/volumes/93/8/14-148338.pdf papers3://publication/doi/10.2471/BLT.14.148338.

22. Wallis L, Reynolds T, Checkett K. AFEM Handbook of Acute and Emergency Care. 2nd edition. Oxford University Press; 2019.

23. Bitter CC, Rice B, Periyanayagam U, Dreifuss B, Hammerstedt H, Nelson SW, et al. What resources are used in emergency departments in rural sub-Saharan Africa? A retrospective analysis of patient care in a district-level hospital in Uganda. BMJ Open. 2018;8:e019024. doi:10.1136/bmjopen-2017019024.

24. Lewis JM, Feasey NA, Rylance J. Aetiology and outcomes of sepsis in adults in sub-Saharan Africa: a systematic review and meta-analysis. Critical Care. 2019;23:212. doi:10.1186/s13054-019-2501-y.

25. World Health Organization. Global report on the epidemiology and burden of sepsis: current evidence, identifying gaps and future directions. 2020.

26. Lewis JM, Abouyannis M, Katha G, Nyirenda M, Chatsika G, Feasey NA, et al. Population Incidence and Mortality of Sepsis in an Urban African Setting, 2013-2016. Clin Infect Dis. doi:10.1093/cid/ciz1119.

27. Rudd KE, Seymour CW, Aluisio AR, Augustin ME, Bagenda DS, Beane A, et al. Association of the Quick Sequential (Sepsis-Related) Organ Failure Assessment (qSOFA) Score With Excess Hospital Mortality in Adults With Suspected Infection in Low- and Middle-Income Countries. JAMA. 2018;319:2202-11. doi:10.1001/jama.2018.6229.

28. Cunningham C, Brysiewicz P, Sepeku A, White L, Murray B, Lobue N, et al. Developing an emergency nursing short course in Tanzania. African Journal of Emergency Medicine. 2017;7:147-50. doi:10.1016/j.afjem.2017.08.002.

29. Kruk ME, Pate M, Mullan Z. Introducing The Lancet Global Health Commission on High-Quality Health Systems in the SDG Era. The Lancet Global Health. 2017;5:e480-1. doi:10.1016/S2214109X(17)30101-8.

30. Andrews B, Muchemwa L, Kelly P, Lakhi S, Heimburger D, Bernard G. Simplified Severe Sepsis Protocol: A Randomized Controlled Trial of Modified Early Goal-Directed Therapy in Zambia. Critical Care Medicine. 2014;42:2315-24. doi:10.1097/CCM.0000000000000541.

31. Silberberg B, Aston S, Boztepe S, Jacob S, Rylance J. Recommendations for fluid management of adults with sepsis in sub-Saharan Africa: a systematic review of guidelines. Critical Care. 2020;24:286. doi:10.1186/s13054-020-02978-4.

\section{Figures}




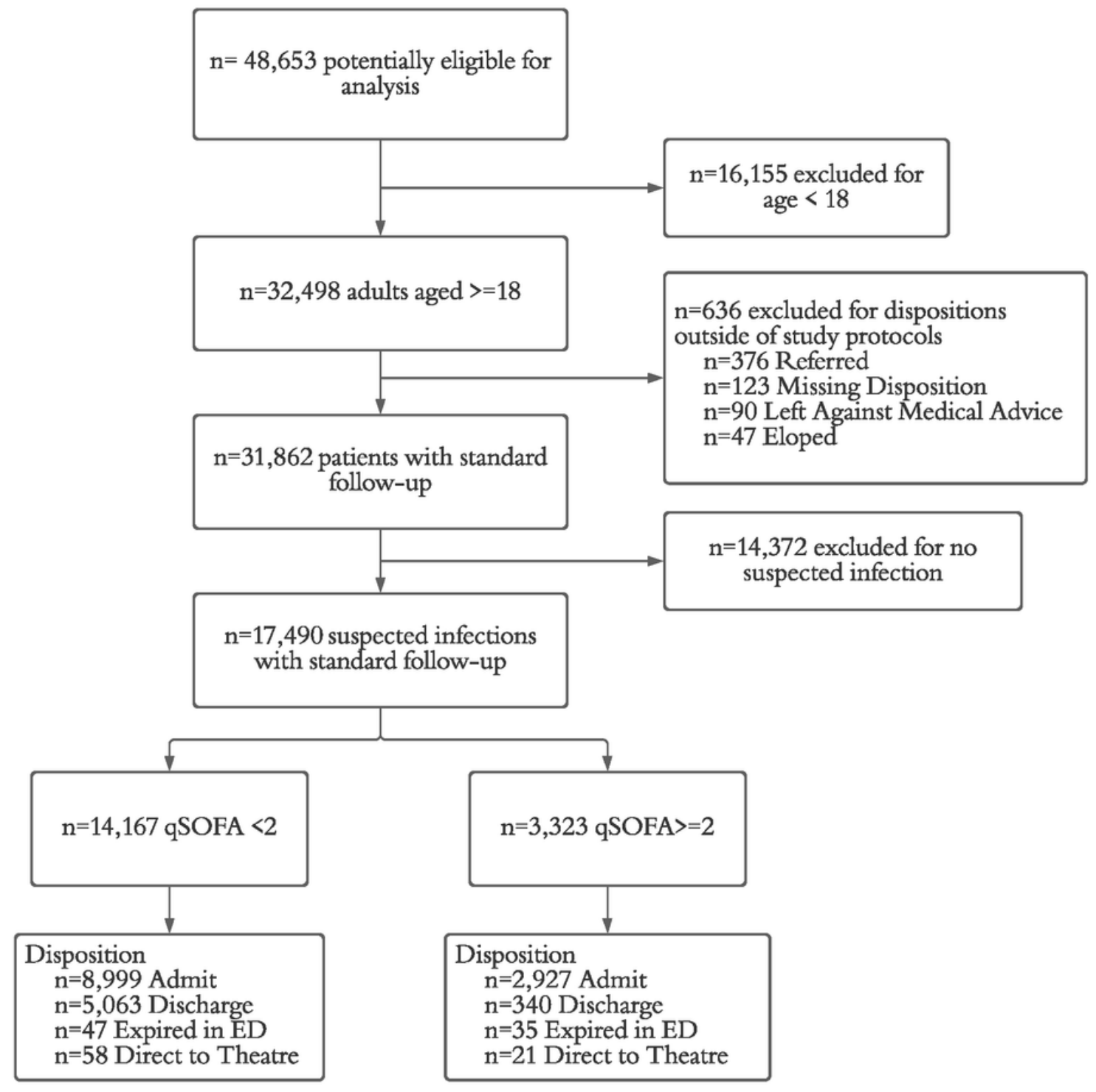

Figure 1

There were 48,653 patient visits from 2010-2019 


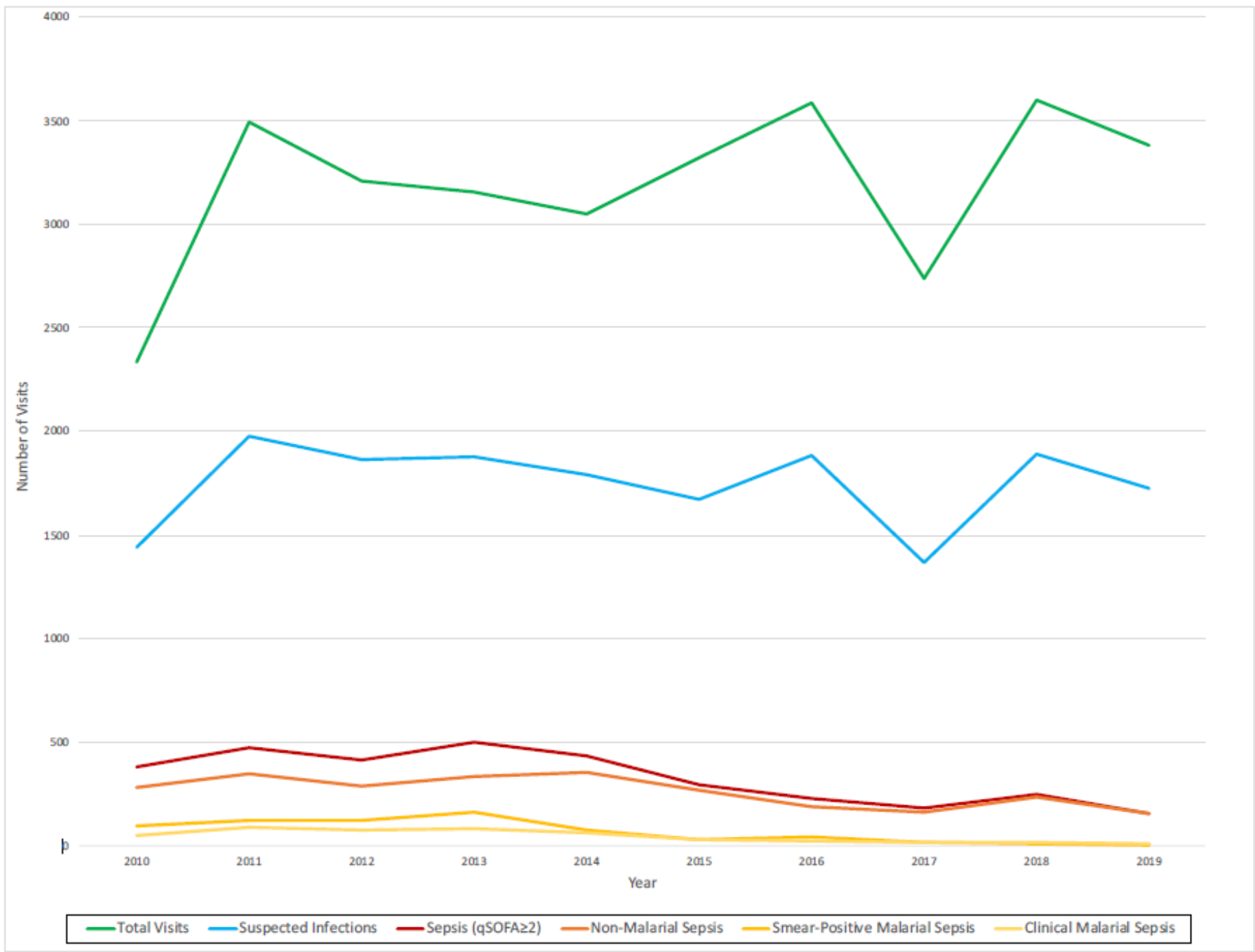

Figure 2

The annual number of visits for all patients, patients with suspected infections and with malarial and non-malarial sepsis 


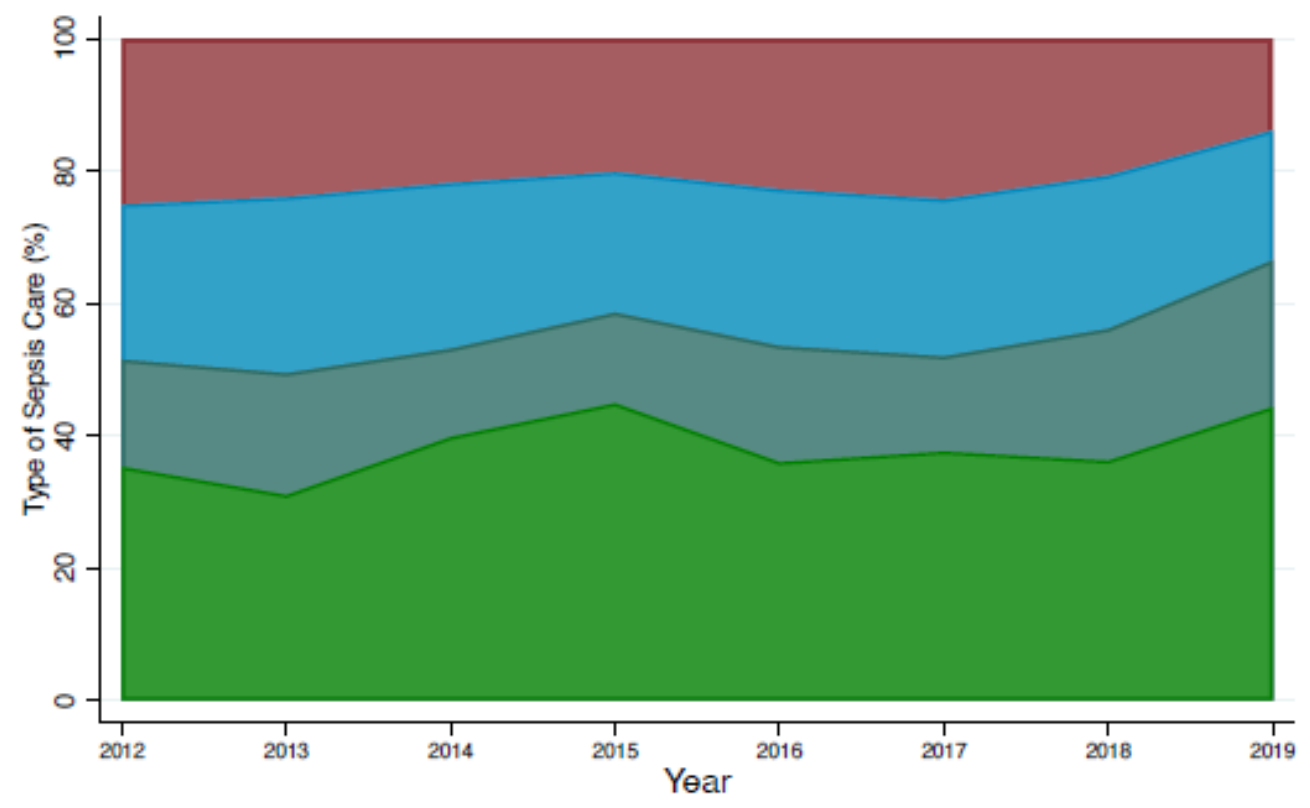

\begin{tabular}{l}
$\square$ Both Fluids \& Anti-Infectives $\quad \square \begin{array}{l}\text { Anti-Infectives Only } \\
\text { Fluids Only }\end{array} \quad \square$ No Fluids or Anti-Infectives \\
\hline
\end{tabular}

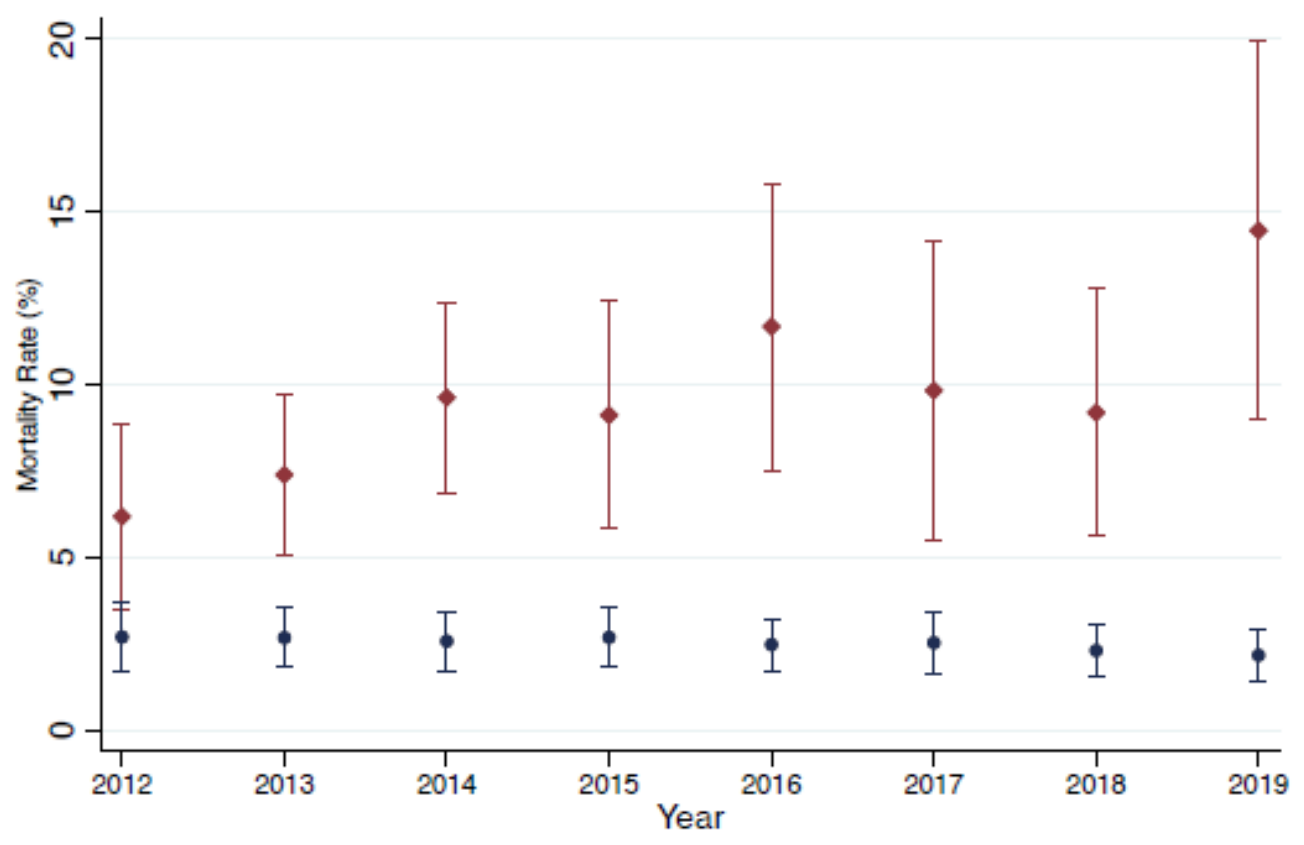

- Sepsis Mortality [95\% Cl] $\quad$ Non-Sepsis Mortality [95\% Cl]

\section{Figure 3}

The annual trends in sepsis management and mortality from 2012-2019. 

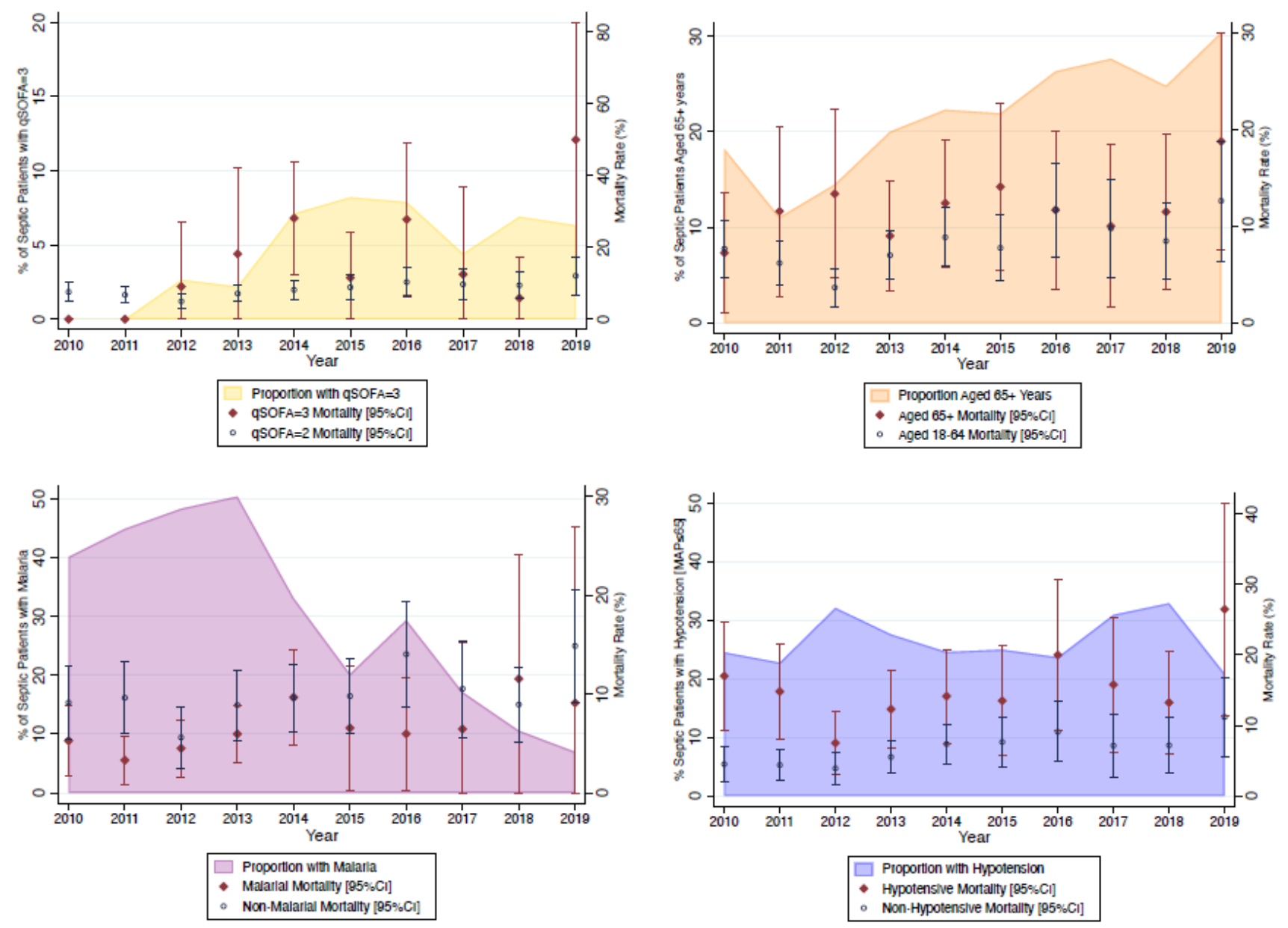

Figure 4

Trends in prevalence and associated mortality for sub-groups of septic patients by year from 2010-2019. 


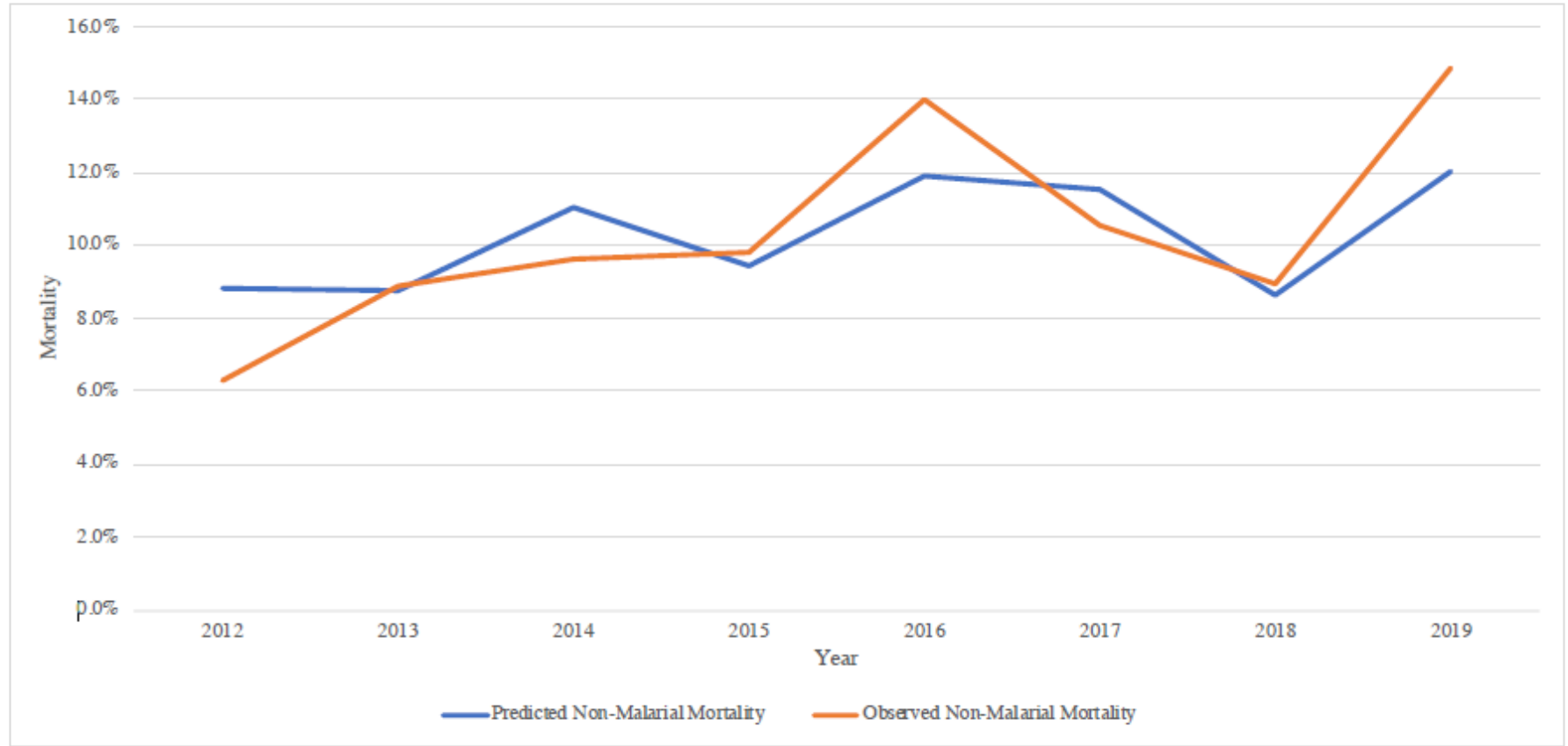

\section{Figure 5}

The predicted mortality coefficients generated by this model were plotted against observed mortality across programme years and both predicted and observed mortality could be seen to increase across the study period

\section{Supplementary Files}

This is a list of supplementary files associated with this preprint. Click to download.

- GraphicalAbstract.png

- Additionalfile1.pdf

- Additionalfile2.pdf 